\title{
Critical appraisal of bevacizumab in the treatment of ovarian cancer
}

\author{
This article was published in the following Dove Press journal: \\ Drug Design, Development and Therapy \\ 28 April 2015 \\ Number of times this article has been viewed
}

\section{Hiroyuki Yoshida \\ Akira Yabuno \\ Keiichi Fujiwara}

Department of Gynecologic

Oncology, Saitama Medical University International Medical Center, Hidaka,

Saitama, Japan
Correspondence: Hiroyuki Yoshida Department of Gynecologic Oncology, Saitama Medical University International Medical Center, I397-I Yamane, Hidaka City, Saitama 350-1298, Japan

Tel +8I 429844637

Fax +8I 42984474 I

Email hiro_y@saitama-med.ac.jp
Abstract: Bevacizumab is the first molecular-targeted agent to be used for the treatment of ovarian cancer. Bevacizumab is a humanized monoclonal antibody targeting vascular endothelial growth factor. Two randomized Phase III trials evaluated the combination of bevacizumab plus standard cytotoxic chemotherapy for first-line treatment of advanced ovarian cancer. Additional Phase III trials evaluated bevacizumab combined with cytotoxic chemotherapy in platinumsensitive and platinum-resistant recurrent ovarian cancer. All these trials reported a statistically significant improvement in progression-free survival but not in overall survival. Furthermore, bevacizumab effectively improved the quality of life with regard to abdominal symptoms in recurrent ovarian cancer patients. Bevacizumab is associated with adverse events not commonly observed with cytotoxic agents used to treat gynecological cancers, such as hypertension, bleeding, thromboembolism, proteinuria, delayed wound healing, and gastrointestinal events. However, most of these events can be adequately managed by gynecologists. The clinical trial results with bevacizumab have supported its recent approval in Europe and the United States as a treatment for ovarian cancer. This review presents the latest evidence for bevacizumab therapy of ovarian cancer and describes selection of patients for personalized treatment.

Keywords: anti-angiogenesis, chemotherapy, biomarkers

\section{Introduction}

Ovarian cancer is known to have the worst prognosis among gynecological malignancies. ${ }^{1}$ Owing to the lack of characteristic symptoms in the early stage and effective screening methods, approximately $60 \%$ patients with ovarian cancer are diagnosed in the advanced stage. ${ }^{1}$ Chemotherapy is the main treatment method for advanced ovarian cancer, and to date, gynecologists have primarily used cytotoxic agents, including platinum, and taxane agents. Bevacizumab (BV) has recently been applied as the first molecular targeting agent for ovarian cancer. BV is a humanized monoclonal antibody, particularly targeting the vascular endothelial growth factor (VEGF). ${ }^{2}$ The VEGF binds to the VEGF receptor (VEGFR) expressed on the cell membrane and promotes cell proliferation, angiogenesis, and vascular hyperpermeability. In particular, BV binds to the VEGF and prevents the VEGF from binding to the VEGFR; as a result, BV exhibits an antitumor effect.

Among the various types of cancers, ovarian cancer is considered to have a high dependence on angiogenic factors during tumor progression. ${ }^{3}$ In fact, it has been reported that the VEGF is overexpressed in most ovarian cancers and correlates with their prognosis. ${ }^{4,5}$ Therefore, based on these characteristics, BV may be much more effective in ovarian cancer than in other cancer types. This review presents the latest evidence supporting the use of BV therapy in ovarian cancer and describes potential personalized treatment for BV therapy. 


\section{BV as first-line therapy}

The Gynecologic Oncology Group (GOG)-0218 trial was a Phase III randomized, double-blind, placebo-controlled trial with three arms that examined the significance of BV in combination with standard chemotherapy using paclitaxel + carboplatin (TC therapy). This study was conducted on 1,873 patients who had not received treatment after surgery for the International Federation of Gynecology and Obstetrics (FIGO) stage III-IV advanced epithelial ovarian cancer, carcinoma of the fallopian tube, or peritoneal cancer. ${ }^{6}$ A comparison was performed with subjects assigned to one of the three groups: the TC therapy plus placebo followed by placebo maintenance group (TCP group) $(n=625)$; TC therapy with concomitant $\mathrm{BV}$ followed by placebo maintenance group (TCBV group) ( $\mathrm{n}=625)$; and TC therapy with concomitant $\mathrm{BV}$ followed by maintenance $\mathrm{BV}$ group (TCBV+ group) $(n=623)$. TC therapy was administered in six cycles every 3 weeks, and BV (or the placebo) was administered from the second cycle every 3 weeks for up to 21 cycles. As shown in Table 1, the primary endpoint of median progressionfree survival (PFS) was 10.3 months in the TCP group and 11.2 months in the TCBV group with no significant difference observed (hazard ratio [HR]: 0.908, 95\% confidence interval [CI]: 0.759-1.040, $P=0.16$ ). However, in the TCBV+ group, the median PFS was 14.1 months, which was significantly longer than that observed in the TCP group (HR: 0.717, 95\% CI: $0.625-0.824, P<0.001)$. Furthermore, in the same trial, an additional analysis was conducted where the progression events based only on CA-125 elevation were not considered as events. As a result, the median PFS was 12.0 months in the TCP group and 18.0 months in the TCBV+ group, with a significant extension of PFS in the TCBV+ group (HR: 0.645, 95\% CI: $0.551-0.756, P<0.001)$. Conversely, for the secondary endpoint, the median overall survival (OS) was 39.3 months in the TCP group and 38.7 months in the TCBV group; the difference was not significant (HR: 1.036, 95\% CI: $0.827-1.297, P=0.76$ ). In the TCBV+ group, the median OS was 39.7 months with no significant difference compared with the TCP group (HR: $0.915,95 \% \mathrm{CI}$ : $0.727-1.15, P=0.45$ ).

The reason for no improvement in OS may be attributed to the fact that crossover (the administration of BV to patients in the TCP group as a treatment in the event of recurrence) was permitted in the present trial. In fact, approximately $40 \%$ of the TCP group received BV following progression. During the present trial, BV was newly listed in the National Comprehensive Cancer Network guidelines as the treatment for recurrent ovarian cancer; therefore, it was deemed difficult to set limitations for the BV crossover of patients in the TCP group. When the crossover was permitted, it was expected that it would be difficult to detect any difference in OS; therefore, during the present trial, the primary endpoint was changed from OS to PFS. This change was implemented based on the consensus that PFS can be used as a surrogate endpoint for OS in the first-line treatment of ovarian cancer at the public workshop of the US Food and Drug Administration (FDA), the American Society of Clinical Oncology, and the American Association for Cancer Research. $^{7}$ Another reason for no improvement in OS that was observed in the present trial was attributed to the long

Table I Summary of Phase III randomized trials of BV in first-line treatment for ovarian cancer

\begin{tabular}{|c|c|c|}
\hline Study & GOG-021 $8^{6}$ & ICON7 ${ }^{10,11}$ \\
\hline Population (n) & $\mathrm{I}, 873$ & $\mathrm{I}, 528$ \\
\hline Eligibility & $\begin{array}{l}\text { Optimal and suboptimal resected stage III } \\
\text { or any stage IV }\end{array}$ & $\begin{array}{l}\text { Stage I-IIA (clear cell, grade 3): } \\
\text { stage IIA-IV }\end{array}$ \\
\hline \multirow[t]{3}{*}{ Regimen } & $\mathrm{TCP}$ & $\mathrm{TC}$ \\
\hline & TCBV & $\mathrm{TCBV}+(7.5)$ \\
\hline & $\mathrm{TCBV}+$ & \\
\hline Dose of BV & $15 \mathrm{mg} / \mathrm{kg}$, triweekly (TCBV: 5 cycles, TCBV+: 21 cycles) & $7.5 \mathrm{mg} / \mathrm{kg}$, triweekly, 18 cycles \\
\hline \multirow[t]{3}{*}{ Median PFS (months) } & TCP: 10.3 & TC: 17.3 \\
\hline & TCBV: II.2 & $\mathrm{TCBV}+(7.5): 19.0$ \\
\hline & TCBV+: 14.1 & \\
\hline \multirow[t]{2}{*}{ HR, $P$-value } & $0.908,0.16^{\mathrm{a}}$ & $0.8 \mathrm{I}, 0.004 \mathrm{I}$ \\
\hline & $0.717,<0.00 I^{b}$ & \\
\hline \multirow[t]{3}{*}{ Median OS (months) } & TCP: 39.3 & TC: 58.6 \\
\hline & TCBV: 38.7 & $\mathrm{TCBV}+(7.5): 58.0$ \\
\hline & $\mathrm{TCBV}+: 39.7$ & \\
\hline \multirow[t]{2}{*}{ HR, $P$-value } & $1.036,0.76^{\mathrm{a}}$ & $0.99,0.85$ \\
\hline & $0.915,0.45^{b}$ & \\
\hline
\end{tabular}

Notes: ${ }^{\mathrm{a}} \mathrm{TCP}$ versus TCBV; ${ }^{\mathrm{T}} \mathrm{TCBV}$ versus $\mathrm{TCBV}+$.

Abbreviations: BV, bevacizumab; HR, hazard ratio; OS, overall survival; PFS, progression-free survival; TC, paclitaxel + carboplatin; TCBV, TC + BV $\rightarrow$ placebo; TCBV+, $\mathrm{TC}+\mathrm{BV} \rightarrow \mathrm{BV} ; \mathrm{TCP}, \mathrm{TC}+$ placebo $\rightarrow$ placebo. 
post-progression survival (PPS) of ovarian cancer patients. The PPS of patients with ovarian cancer is usually longer than that of those with other solid tumors because there are many post-progression therapies. The significant difference of PFS may be lost in the noise of post-progression therapies. In the event of a disease with a long PPS, a very large sample size is required for the prolonged PFS to be reflected in OS; therefore, it was difficult to show the significant difference in OS, although it was obtained in PFS. ${ }^{8}$ In the present study, PPS was considered to be more than 20 months long, which made it difficult for the significant difference observed for PFS to be reflected in OS.

Furthermore, quality of life (QOL) was evaluated in the present trial. Because there was no significant extension of OS observed, the question whether BV treatment improved QOL is of strong significance. QOL was evaluated using the Trial Outcome Index of the Functional Assessment of Cancer Therapy-Ovary. Although QOL of the TCBV and TCBV+ groups was lower than that of the TCP group before cycle 4 and cycle 7 , there was no significant difference between the three groups during maintenance phase. ${ }^{6,9}$

The International Cooperative Group for Ovarian Neoplasia (ICON)7 trial was a Phase III randomized, double-blind trial with two arms that examined the significance of BV in combination with TC therapy. ${ }^{10,11}$ This study was performed on 1,528 patients who had not received treatment after surgery for FIGO stage I-IIA (clear cell histology or grade 3) and stage IIB-IV epithelial ovarian cancer, carcinoma of the fallopian tube, or peritoneal cancer. Patients were assigned to one of the two groups, including the standard chemotherapy group administered six cycles of TC therapy every 3 weeks (TC group) ( $\mathrm{n}=764)$, and TC therapy with concomitant BV followed by maintenance BV administered for 12 additional cycles every 3 weeks (TCBV+ (7.5) group) $(n=764)$. There was no placebo, and the BV dose used was $7.5 \mathrm{mg} / \mathrm{kg}$ rather than the $15 \mathrm{mg} / \mathrm{kg}$ used in the GOG-0218 trial. As shown in Table 1, median PFS was 17.3 months in the TC group and 19.0 months in the TCBV+ (7.5) group (HR: $0.81,95 \% \mathrm{CI}$ : $0.70-0.94, P=0.0041)$, indicating that PFS was significantly prolonged with additional $\mathrm{BV}$. In general, post-progression crossover to BV was not permitted, and for the secondary endpoint, the mean OS was 58 months in both groups with no significant difference observed, similar to that in the GOG-0218 trial (HR: 0.99, 95\% CI: 0.85-1.14, $P=0.85$ ). ${ }^{11}$ Furthermore, QOL was evaluated using the European Organization for Research and Treatment of Cancer Quality of Life Questionnaire-Ovarian Cancer Module 28 (EORTC QLQ-OV28) and the EORTC QLQ-core 30, and a reduction in QOL was found in the TCBV+ (7.5) group. ${ }^{12}$
In both the trials, although there was no significant difference observed in OS, notable results were found on subgroup analysis. In the subgroup analysis of the GOG-0218 trial, when subjects were limited to stage IV patients, the median OS was 32.8 months in the TCP group in contrast to 40.6 months in the TCBV+ group, which shows a prolongation of 7.8 months (HR: $0.72,95 \% \mathrm{CI}$ : 0.53-0.97). ${ }^{13,14}$ Furthermore, in the ICON7 trial, when subjects were limited to high-risk patients (stage IV or stage III with more than $1 \mathrm{~cm}$ residual tumor after surgery), the median OS was 30.3 months in the TC group and 39.7 months in the $\mathrm{TCBV}+(7.5)$ group, indicating a significant prolongation of 9 months with BV treatment (HR: 0.64, 95\% CI: 0.48-0.85, $P=0.002) .{ }^{10,11}$ However, it should be noted that these results were based on the exploratory analysis.

Based on the results of the aforementioned two clinical trials, on examining the use of BV as first-line chemotherapy to treat patients with ovarian cancer, the treatment may be considered to be appropriate for high-risk patients such as stage III with residual disease or stage IV. In patients with stage I and II, the indication of BV administration should be determined after thoroughly examining the risks and benefits in terms of the likelihood of recurrence, the side effects of BV treatment, and its cost-effectiveness. However, attention should be paid to the fact that the significant extension of OS in high-risk patients was the results of subgroup analysis in which the consistency of patient characteristics was not ensured. Furthermore, the efficacy of the two doses ( $7.5 \mathrm{mg} / \mathrm{kg}$ or $15 \mathrm{mg} / \mathrm{kg}$ ) of BV for ovarian cancer patients is under consideration. Given that there was no prolongation in OS observed in the ICON7 trial in which there was no crossover to $\mathrm{BV}$, we may not expect that OS would be prolonged with first-line BV treatment at a dose of $7.5 \mathrm{mg} / \mathrm{kg}$. On the other hand, adverse events tend to be more common at a dose of $15 \mathrm{mg} / \mathrm{kg}$ than $7.5 \mathrm{mg} / \mathrm{kg} .{ }^{15}$ This is a problem that cannot be immediately addressed; however, considering the difference of the efficacy in the GOG-0218 and ICON7 trials and cost-effectiveness, the higher dose of $15 \mathrm{mg} / \mathrm{kg}$ may be unnecessary.

\section{BV in the treatment of recurrent ovarian cancer}

The OCEANS trial was a Phase III randomized, double-blind, placebo-controlled trial on 484 patients with the primary recurrence of platinum-sensitive advanced epithelial ovarian cancer, peritoneal cancer, and carcinoma of the fallopian tube (Table 2). ${ }^{16}$ Patients were randomly assigned to either the gemcitabine + carboplatin therapy (GC therapy) in combination with placebo group (GCP group) or the GC therapy with 
Table 2 Summary of Phase III randomized trials of BV in recurrent ovarian cancer

\begin{tabular}{|c|c|c|}
\hline Study & OCEANS ${ }^{16,17}$ & AURELIA ${ }^{18}$ \\
\hline Population (n) & 484 & 361 \\
\hline Eligibility & Platinum-sensitive recurrence (PFI $>6$ months) & Platinum-resistant recurrence (PFI $\leq 6$ months) \\
\hline \multirow[t]{2}{*}{ Regimen } & $\mathrm{GCP}$ & $\mathrm{CT}^{\mathrm{a}}$ \\
\hline & $\mathrm{GCBV}+$ & CTBV \\
\hline Dose of BV & $15 \mathrm{mg} / \mathrm{kg}$, triweekly, until progression & 15 mg/kg, triweekly, until progression \\
\hline \multirow[t]{2}{*}{ Median PFS (months) } & GCP: 8.4 & CT: 3.4 \\
\hline & GCBV+: 12.4 & CTBV: 6.7 \\
\hline HR, $P$-value & $0.484,<0.001$ & $0.48,<0.001$ \\
\hline \multirow[t]{2}{*}{ Median OS (months) } & GCP: 33.7 & CT: 13.3 \\
\hline & GCBV+: 33.4 & CTBV: 16.6 \\
\hline HR, $P$-value & $0.960,0.736$ & $0.85,0.17$ \\
\hline \multirow[t]{2}{*}{ ORR (\%) } & GCP: 57.4 & CT: 12.6 \\
\hline & GCBV+: 78.5 & CTBV: 30.9 \\
\hline$P$-value & $<0.001$ & $<0.001$ \\
\hline
\end{tabular}

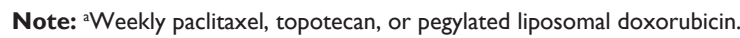

Abbreviations: BV, bevacizumab; CT, chemotherapy; CTBV, CT + BV; GCBV+, gemcitabine + carboplatin + BV $\rightarrow$ BV; GCP, gemcitabine + carboplatin + placebo $\rightarrow$ placebo; $\mathrm{HR}$, hazard ratio; ORR, objective response rate; OS, overall survival; PFI, platinum-free interval; PFS, progression-free survival.

concomitant BV followed by BV treatment until progression group (GCBV+ group). As the primary endpoint, median PFS was 8.4 months in the GCP group as compared with 12.4 months in the GCBV+ group (HR: $0.484,95 \%$ CI: $0.388-$ $0.605, P<0.001)$ with a significant prolongation observed in the GCBV+ group. The objective response rate was $57.4 \%$ in the GCP group, whereas it was $78.5 \%$ in the GCBV+ group with a significant difference shown $(P<0.001)$. On the other hand, the median OS was 33.7 months in the GCP group and 33.4 months in the GCBV+ group (HR: 0.960, 95\% CI: $0.760-1.214, P=0.736$ ) with no significant difference observed. ${ }^{17}$ The reason for no significant difference in OS observed could be attributed to BV crossover as seen in the GOG-0218 trial. Furthermore, in this trial, we also noted that PPS was 2 years long; this may have led to the 4-month difference observed in PFS not being reflected in OS.

The AURELIA trial was a Phase III randomized, double-blind trial on 361 patients with recurrent platinumresistant epithelial ovarian cancer, peritoneal cancer, and carcinoma of the fallopian tube, whose disease had progressed $\leq 6$ months after receiving four cycles or more of treatment with platinum-based chemotherapy (Table 2). ${ }^{18}$ Patients were randomly assigned to the single-agent therapy group (CT group) or the chemotherapy plus BV treatment group (CTBV group). Chemotherapy was chosen by investigators from three agents, including pegylated liposomal doxorubicin (PLD) $(n=126)$, topotecan $(n=120)$, and weekly paclitaxel $(n=115)$. Furthermore, pretreatment history was limited to two regimens. As the primary endpoint, median PFS was 3.4 months in the CT group in contrast to 6.7 months in the CTBV group (HR: 0.48, 95\%
CI: $0.38-0.60, P<0.001$ ), with significantly better results in the CTBV group. For all three agents, including PLD, topotecan, and weekly paclitaxel, results were significantly better when administered in combination with BV. The objective response rate was $12.6 \%$ in the CT group and $30.9 \%$ in the CTBV group with a significant difference observed $(P<0.001)$. On the other hand, the median OS was 13.3 months in the CT group and 16.6 months in the CTBV group (HR: $0.85,95 \%$ CI: $0.66-1.08, P=0.17$ ) with no significant difference observed. The reason for no significant difference found in OS was assumed to be due to crossover to $\mathrm{BV}$ in the trial. Although there has been no significant prolongation in OS to date, the response rate for platinum-resistant ovarian cancer was 10\%-15\% in single-agent chemotherapy and approximately $20 \%$ in combination therapy. Thus, the results of this trial indicated a response rate of more than $30 \%$, and significant prolongation of PFS strongly indicated that BV may be an option for treating platinum-resistant ovarian cancer. Furthermore, in the AURELIA trial, QOL was examined as the secondary endpoint. Surveys were initiated prior to the treatment using the EORTC QLQ-OV28 and Functional Assessment of Cancer Therapy-Ovarian Cancer Symptom Index, and were performed every two to three courses. As a result, 8-9 weeks after the start of treatment, the rate of patients who showed a $15 \%$ improvement in abdominal and gastrointestinal symptoms was $12.7 \%$ greater in the CTBV group than in the CT group (21.9\% versus $9.3 \%, 95 \% \mathrm{CI}$ : 4.4-20.9, $P=0.002) .{ }^{19}$

The results of the aforementioned two clinical trials of recurrent ovarian cancer demonstrate the effectiveness of 
BV in recurrent ovarian cancer. Notably, in the AURELIA trial of platinum-resistant ovarian cancer, the significant extension of PFS and response rate of over 30\% were muchappreciated results, as there are few treatment options for platinum-resistant ovarian cancer. Furthermore, although the AURELIA trial was not a placebo-controlled trial, the improved QOL with regard to abdominal symptoms in the $\mathrm{BV}$ combination therapy group was considered to be a result of extreme clinical importance, because the patients with recurrent ovarian cancer often present with difficult-to-treat abdominal symptoms, including abdominal pain, ascites, and constipation. Along with the results of the AURELIA trial, FDA approved BV in combination with PLD, topotecan, or paclitaxel for the treatment of patients with platinum-resistant recurrent ovarian, fallopian tube, or peritoneal cancer who received no more than two prior chemotherapy regimens.

\section{Treatment-related toxicity}

Adverse events characteristic to BV include hypertension, bleeding, thromboembolism, proteinuria, delayed wound healing, and gastrointestinal events (Table 3). In particular, gastrointestinal events, such as perforations or fistulae, are an adverse event that requires utmost attention, with an incidence in the GOG-0218 trial of $1.2 \%$ in the TCP group, $2.8 \%$ in the TCBV group, and $2.6 \%$ in the TCBV+ group. ${ }^{6}$ In the ICON7 trial, the incidence was $0.4 \%$ in the TC group and $1.3 \%$ in the TCBV+ group. ${ }^{10}$ On the other hand, there was no gastrointestinal event observed in the OCEANS trial and a low incidence of $2.2 \%$ in the CTBV group in the AURELIA trial. ${ }^{16,17}$ This may be attributed to the fact that, in the OCEANS trial, patients had few pretreatments with only one to two regimens, whereas in the AURELIA trial, pretreatment history was limited to two regimens and patients with a high risk of gastrointestinal tract perforation, such as intestinal obstruction, were excluded from enrollment. The patient selection for the OCEANS and AURELIA trials was based on the result of the AVF2949g trial. In the AVF2949g trial, BV was administered as a single agent to treat patients with platinum-resistant ovarian cancer, whose prior treatment regimens were no more than three. In this trial, gastrointestinal tract perforation occurred in five of 44 patients $(11.4 \%)$. When patients were limited to those with a pretreatment history of three regimens, gastrointestinal tract perforation occurred in five of 21 patients $(23.8 \%)$, indicating that a pretreatment history of three regimens was a significant risk factor for gastrointestinal tract perforation. ${ }^{20}$ Based on the above data, to prevent gastrointestinal tract perforation in BV treatment, the number of previous chemotherapy regimens, patient abdominal symptoms, and intraperitoneal conditions examined using diagnostic imaging should be thoroughly considered before BV administration. Moreover, it is assumed that, in the overall treatment plan, administering $\mathrm{BV}$ as early as possible will reduce the risk of gastrointestinal tract perforation. Although adverse events of BV also include events not commonly observed in cytotoxic agents used by gynecologists to date, they can be adequately managed by gynecologists. If administered with due attention to severe adverse events such as gastrointestinal tract perforation, BV can be used safely.

\section{Predictive biomarkers for BV treatment}

Molecular targeting agents, including BV, exert their therapeutic effect by targeting specific molecules. Therefore, no therapeutic effect can be anticipated unless the cancer cells express the target molecules. Research into predictive biomarkers for BV treatment is underway, and it is assumed that VEGFR-1 and neuropilin-1 found in the plasma or cancer tissue are potential candidates for predictive biomarkers. ${ }^{21}$ Recently, Steffensen et al reported that cell-free DNA in

Table 3 Adverse events occurring in randomized Phase III trials of BV in advanced ovarian cancer

\begin{tabular}{|c|c|c|c|c|c|c|c|c|c|}
\hline \multirow[t]{2}{*}{ Adverse events (\%) } & \multicolumn{3}{|c|}{ GOG-021 $8^{6}$} & \multicolumn{2}{|c|}{ ICON7 $^{10}$} & \multicolumn{2}{|c|}{ OCEANS $^{16}$} & \multicolumn{2}{|c|}{ AURELIA ${ }^{18}$} \\
\hline & TCP & TCBV & TCBV+ & TC & TCBV+ & GCP & GCBV+ & CT & СTBV \\
\hline Gl events (grade $\geq 2$ ) & 1.2 & 2.8 & 2.6 & 0.4 & 1.3 & 0 & 0 & 0 & 2.2 \\
\hline Hypertension (grade $\geq 2$ ) & 7.2 & 16.5 & 22.9 & 2.1 & 18.3 & 0.4 & 17.4 & 6.6 & 20.1 \\
\hline VTE $($ grade $\geq 3)$ & 5.8 & 5.3 & 6.7 & 4.1 & 6.7 & 2.6 & 4.0 & 4.4 & 2.8 \\
\hline Proteinuria (grade $\geq 3$ ) & 0.7 & 0.7 & 1.6 & 0.1 & 0.5 & 0.9 & 8.5 & 0.6 & 10.6 \\
\hline $\begin{array}{l}\text { Delayed wound healing } \\
\text { (grade } \geq 3 \text { ) }\end{array}$ & 2.8 & 3.6 & 3.0 & 2.1 & 5.0 & 0 & 0.8 & 0 & 0 \\
\hline $\begin{array}{l}\text { Non-CNS bleeding } \\
\text { (grade } \geq 3 \text { ) }\end{array}$ & 0.8 & 1.3 & 2.1 & 0.3 & I & 0.9 & 5.7 & 0.6 & 0.6 \\
\hline
\end{tabular}

Abbreviations: BV, bevacizumab; CT, chemotherapy; CNS, central nervous system; CTBV, CT + BV; GCBV+, gemcitabine + carboplatin + BV $\rightarrow$ BV; GCP, gemcitabine + carboplatin + placebo $\rightarrow$ placebo; Gl, gastrointestinal; TC, paclitaxel + carboplatin;TCBV,TC + BV $\rightarrow$ placebo; TCBV+,TC + BV $\rightarrow$ BV; TCP,TC + placebo $\rightarrow$ placebo; VTE, venous thromboembolism. 
the plasma is a promising predictive biomarker for singleagent BV treatment. ${ }^{22}$ Furthermore, the cases of high-grade serous ovarian cancer in the ICON7 trial were divided into the immunogenic group with an accelerated expression of immune-related genes and the angiogenic group with an accelerated expression of angiogenesis-related genes, and the effects of BV in each group were compared. As a result, the prognosis of the immunogenic group was significantly poor with combination BV therapy, whereas in the angiogenic group, PFS was prolonged with combination BV therapy. ${ }^{23}$ On the other hand, a comprehensive gene analysis conducted in the Cancer Genome Atlas found that high-grade serous ovarian cancer can be divided into four different subtypes, including differentiated, proliferative, mesenchymal, and immunoreactive subtypes. ${ }^{24,25}$ The difference in the effectiveness of BV was examined in patients in the ICON7 trial according to these four subtypes. ${ }^{26}$ As a result, a marked therapeutic effect was seen in the proliferative and mesenchymal subtype, whereas in the differentiated and immunoreactive types, the effect was poor. The mechanism underlying the difference in the effectiveness of BV in the molecular subtypes of ovarian cancer has not been fully understood and further studies are warranted. However, there are high hopes for biomarkers capable of accurately predicting the therapeutic effect of $\mathrm{BV}$, as this would enable more effective administration of BV for ovarian cancer patients.

\section{Prospects for the future}

Several Phase III clinical trials using BV to treat ovarian cancer are under way (Table 4). One of these is the BOOST trial, which examines the optimal administration period of $\mathrm{BV} .{ }^{27}$ The effectiveness of $\mathrm{BV}$ in the first-line treatment for ovarian cancer can be achieved by adding the maintenance therapy; however, the optimal administration period is unknown. In this trial, patients who received BV in combination with TC therapy followed by BV maintenance therapy were divided into two groups: the group administered $\mathrm{BV}$ for a total of 15 months and the group administered BV for 30 months. Furthermore, concerning recurrent patients who received combination $\mathrm{BV}$ therapy as the first-line treatment, the MITO-16/MANGO2b trial currently under way examines whether chemotherapy in combination with BV remains effective. ${ }^{28}$ In addition, the GOG-0252 trial currently under way examines the effect of the intraperitoneal administration of carboplatin and weekly administration of paclitaxel in combination with BV. ${ }^{29}$ However, to date, only the OCEANS trial has used GC therapy in combination with $\mathrm{BV}$ to treat platinum-sensitive patients with recurrent ovarian cancer. The GOG-0213 trial examines the effectiveness of TC therapy in combination with BV for treating platinumsensitive patients with recurrent ovarian cancer. ${ }^{30} \mathrm{We}$ believe that the results of these trials will further clarify the role of $\mathrm{BV}$ in the treatment of ovarian cancer.

\section{Conclusion}

This article provides the latest evidence and predicted further developments in BV treatment for ovarian cancer. BV is the first molecular targeting agent to be indicated for gynecological cancers, and, based on the specific tumor characteristics of ovarian cancer, BV could be potentially much more effective for ovarian cancer than for other cancer types. As a result of the four clinical trials described above, in the treatment of both primary and recurrent cancers, BV is believed to prolong the disease control of ovarian cancer and is a very promising agent. ${ }^{15,31}$ Although adverse events of BV contained events not commonly observed with cytotoxic agents used to treat ovarian cancer, these events can be adequately managed with careful attention to severe adverse events such as gastrointestinal tract perforation. Furthermore, in recurrent ovarian cancer patients who present with various difficult-to-treat

Table 4 Summary of randomized Phase III trials currently ongoing

\begin{tabular}{|c|c|c|c|c|}
\hline Study & BOOST $^{27}$ & MITO-I6/MANGO2b ${ }^{28}$ & GOG-0252 29 & GOG-O21 $3^{30}$ \\
\hline Eligibility & $\begin{array}{l}\text { Newly diagnosed } \\
\text { stage IIB-IV }\end{array}$ & $\begin{array}{l}\text { Recurrence at least } 6 \text { months } \\
\text { after front-line } T C+B V \rightarrow B V\end{array}$ & $\begin{array}{l}\text { Optimal or suboptimal resected } \\
\text { stage II-IV }\end{array}$ & $\begin{array}{l}\text { Platinum-sensitive } \\
\text { recurrence (PFI }>6 \text { months) }\end{array}$ \\
\hline Regimen & $\begin{array}{l}\mathrm{TC}+\mathrm{BV} \rightarrow \mathrm{BV} \text { (total } 22 \\
\text { cycles) versus } \mathrm{TC}+\mathrm{BV} \rightarrow \\
\mathrm{BV} \text { (total } 44 \text { cycles) }\end{array}$ & $\begin{array}{l}\mathrm{CT} \text { versus } \mathrm{CT}+\mathrm{BV} \rightarrow \mathrm{BV} \\
\text { (until progression) }\end{array}$ & $\begin{array}{l}\text { ddTCiv }+ \text { BV } \rightarrow \text { BV (total } 2 \text { I } \\
\text { cycles) versus ddTCip }+ \text { BV } \rightarrow \text { BV } \\
\text { (total } 2 \text { I cycles) versus ddTPip }+ \\
\text { BV } \rightarrow \text { BV (total } 2 \text { I cycles) }\end{array}$ & $\begin{array}{l}\mathrm{TC} \text { versus } \mathrm{TC}+\mathrm{BV} \rightarrow \mathrm{BV} \\
\text { (until progression) }\end{array}$ \\
\hline $\begin{array}{l}\text { Primary } \\
\text { endpoint }\end{array}$ & PFS & PFS & PFS & OS \\
\hline
\end{tabular}

Notes: Pegylated liposomal doxorubicin + carboplatin, gemcitabine + carboplatin, or paclitaxel + carboplatin. Paclitaxel iv on days I, 8, and I5 and carboplatin iv on day I. Treatment repeats every 21 days. Paclitaxel iv on days I, 8 , and 15 and carboplatin ip on day I. Treatment repeats every 21 days. Paclitaxel iv on day I, cisplatin ip on day 2 , and paclitaxel ip on day 8 . Treatment repeats every 21 days.

Abbreviations: BV, bevacizumab; CT, chemotherapy; ddTCiv, dose dense paclitaxel + carboplatin, intravenous; ddTCip, dose dense paclitaxel + carboplatin, intraperitoneal; ddTPip, dose dense paclitaxel + cisplatin, intraperitoneal; ip, intraperitoneal; iv, intravenous; OS, overall survival; PFI, platinum-free interval; PFS, progression-free survival; TC, paclitaxel + carboplatin. 
abdominal symptoms, BV effectively improves QOL with regard to abdominal symptoms, which is a very important clinical finding. However, the improvement in QOL should be confirmed in the future through a placebo-controlled trial. Although BV is effective for ovarian cancer, currently BV treatment has not prolonged OS for either primary or recurrent cancer, and we believe that, given the high cost associated with the treatment, ${ }^{32}$ patients who receive BV treatment should be carefully selected. Therefore, the establishment of biomarkers that predict the effectiveness of BV will be an important factor in determining the importance of BV in the future treatment of ovarian cancer.

\section{Disclosure}

The authors report no conflicts of interest in this work.

\section{References}

1. Siegel R, Naishadham D, Jemal A. Cancer statistics, 2013. CA Cancer J Clin. 2013;63(1):11-30.

2. Ferrara N, Hillan KJ, Novotny W. Bevacizumab (Avastin), a humanized anti-VEGF monoclonal antibody for cancer therapy. Biochem Biophys Res Commun. 2005;333(2):328-335.

3. Hazelton D, Nicosia RF, Nicosia SV. Vascular endothelial growth factor levels in ovarian cyst fluid correlate with malignancy. Clin Cancer Res. 1999;5(4):823-829.

4. Bandiera E, Franceschini R, Specchia C, et al. Prognostic significance of vascular endothelial growth factor serum determination in women with ovarian cancer. ISRN Obstet Gynecol. 2012;2012:245756.

5. Yamamoto S, Konishi I, Mandai M, et al. Expression of vascular endothelial growth factor (VEGF) in epithelial ovarian neoplasms: correlation with clinicopathology and patient survival, and analysis of serum VEGF levels. Br J Cancer. 1997;76(9):1221-1227.

6. Burger RA, Brady MF, Bookman MA, et al; Gynecologic Oncology Group. Incorporation of bevacizumab in the primary treatment of ovarian cancer. $N$ Engl J Med. 2011;365(26):2473-2483.

7. Bast RC, Thigpen JT, Arbuck SG, et al. Clinical trial endpoints in ovarian cancer: report of an FDA/ASCO/AACR Public Workshop. Gynecol Oncol. 2007;107(2):173-176.

8. Broglio KR, Berry DA. Detecting an overall survival benefit that is derived from progression-free survival. J Natl Cancer Inst. 2009; 101(23):1642-1649.

9. Monk BJ, Huang HQ, Burger RA, et al. Patient reported outcomes of a randomized, placebo-controlled trial of bevacizumab in the front-line treatment of ovarian cancer: a Gynecologic Oncology Group Study. Gynecol Oncol. 2013;128(3):573-578.

10. Perren TJ, Swart AM, Pfisterer J, et al; ICON7 Investigators. A phase 3 trial of bevacizumab in ovarian cancer. $N$ Engl J Med. 2011; 365(26):2484-2496.

11. Oza AM, Perren TJ, Swart AM, et al. ICON7: final overall survival results in the GCIG phase III randomized trial of bevacizumab in women with newly diagnosed ovarian cancer [abstract]. E J Cancer. 2013;49(2):LBA:6.

12. Stark D, Nankivell M, Pujade-Lauraine E, et al. Standard chemotherapy with or without bevacizumab in advanced ovarian cancer: quality-of-life outcomes from the International Collaboration on Ovarian Neoplasms (ICON7) phase 3 randomised trial. Lancet Oncol. 2013;14(3):236-243.

13. Randall LM, Burger RA, Nguyen H, et al. Outcome differences in patients with advanced epithelial ovarian, primary peritoneal and fallopian tube cancers treated with and without bevacizumab. Gynecol Oncol. 2013;130(1):e33-e34.
14. Coleman RL, Burger RA, Bookman MA, et al. Analysis of survivorship in high risk patients treated on Gynecologic Oncology Group 218 [abstract]. Gynecol Oncol. 2013;Suppl: S106; SGO \#267.

15. Zhou M, Yu P, Qu X, Liu Y, Zhang J. Phase III trials of standard chemotherapy with or without bevacizumab for ovarian cancer: a meta-analysis. PLoS One. 2013;8(12):e81858.

16. Aghajanian C, Blank SV, Goff BA, et al. OCEANS: a randomized, double-blind, placebo-controlled phase III trial of chemotherapy with or without bevacizumab in patients with platinum-sensitive recurrent epithelial ovarian, primary peritoneal, or fallopian tube cancer. J Clin Oncol. 2012;30(17):2039-2045.

17. Aghajanian C, Nycum LR, Goff B, Nguyen H, Husain A, Blank SV. Updated overall survival analysis in OCEANS, a randomized phase 3 trial of gemcitabine $(\mathrm{G})+$ carboplatin $(\mathrm{C})$ and bevacizumab $(\mathrm{BV})$ or placebo (PL) followed by BV or PL in platinum-sensitive recurrent epithelial ovarian (ROC), primary peritoneal (PPC), or fallopian tube cancer (FTC) [abstract]. Presented at: the ESMO Congress; 2012. Abstract 9670.

18. Pujade-Lauraine E, Hilpert F, Weber B, et al. Bevacizumab combined with chemotherapy for platinum-resistant recurrent ovarian cancer: the AURELIA open-label randomized phase III trial. J Clin Oncol. 2014; 32(13):1302-1308.

19. Stockler MR, Hilpert F, Friedlander M, et al. Patient-reported outcome results from the open-label phase III AURELIA trial evaluating bevacizumab-containing therapy for platinum-resistant ovarian cancer. J Clin Oncol. 2014;32(13):1309-1316.

20. Cannistra SA, Matulonis UA, Penson RT, et al. Phase II study of bevacizumab in patients with platinum-resistant ovarian cancer or peritoneal serous cancer. J Clin Oncol. 2007;25(33):5180-5186.

21. Lambrechts D, Lenz HJ, de Haas S, Carmeliet P, Scherer SJ. Markers of response for the antiangiogenic agent bevacizumab. J Clin Oncol. 2013;31(9):1219-1230.

22. Steffensen KD, Madsen CV, Andersen RF, Waldstrøm M, Adimi P, Jakobsen A. Prognostic importance of cell-free DNA in chemotherapy resistant ovarian cancer treated with bevacizumab. Eur J Cancer. 2014;50(15):2611-2618.

23. Gourley C, McCavigan A, Perren T, et al. Molecular subgroup of highgrade serous ovarian cancer (HGSOC) as a predictor of outcome following bevacizumab. J Clin Oncol. 2014;32:5s (suppl; abstr 5502).

24. Cancer Genome Atlas Research Network. Integrated genomic analyses of ovarian carcinoma. Nature. 2011;474(7353):609-615.

25. Konecny GE, Wang C, Hamidi H, et al. Prognostic and therapeutic relevance of molecular subtypes in high-grade serous ovarian cancer. J Natl Cancer Inst. 2014;106(10).

26. Winterhoff BJN, Kommoss S, Oberg AL, et al. Bevacizumab and improvement of progression-free survival (PFS) for patients with the mesenchymal molecular subtype of ovarian cancer. J Clin Oncol. 2014;32:5s (suppl; abstr 5509).

27. AGO Study Group: Evaluation of optimal initial treatment duration of bevacizumab in combination with standard chemotherapy in patients with ovarian cancer (BOOST). ClinicalTrials.gov identifier: NCT01462890.

28. National Cancer Institute, Naples: Bevacizumab beyond progression in platinum sensitive ovarian cancer (MITO16/MANGO2b). ClinicalTrials.gov identifier: NCT01802749.

29. National Cancer Institute: Bevacizumab and intravenous or intraperitoneal chemotherapy in treating patients with stage II-III ovarian epithelial cancer, fallopian tube cancer, or primary peritoneal cancer. ClinicalTrials.gov identifier: NCT00951496.

30. National Cancer Institute: Carboplatin, paclitaxel and gemcitabine hydrochloride with or without bevacizumab after surgery in treating patients with recurrent ovarian epithelial cancer, primary peritoneal cavity cancer, or fallopian tube cancer. ClinicalTrials.gov identifier: NCT00565851.

31. Ye Q, Chen HL. Bevacizumab in the treatment of ovarian cancer: a meta-analysis from four phase III randomized controlled trials. Arch Gynecol Obstet. 2013;288(3):655-666.

32. Barnett JC, Alvarez Secord A, Cohn DE, Leath CA 3rd, Myers ER, Havrilesky LJ. Cost effectiveness of alternative strategies for incorporating bevacizumab into the primary treatment of ovarian cancer. Cancer. 2013;119(20):3653-3661. 


\section{Publish your work in this journal}

Drug Design, Development and Therapy is an international, peerreviewed open-access journal that spans the spectrum of drug design and development through to clinical applications. Clinical outcomes, patient safety, and programs for the development and effective, safe, and sustained use of medicines are a feature of the journal, which

has also been accepted for indexing on PubMed Central. The manuscript management system is completely online and includes a very quick and fair peer-review system, which is all easy to use. Visit http://www.dovepress.com/testimonials.php to read real quotes from published authors.

Submit your manuscript here: http://www.dovepress.com/drug-design-development-and-therapy-journal 